\title{
Complex Causal Structures of Neighbourhood Change: Evidence From a Functionalist Model and Yelp Data
}

\author{
${ }^{\infty}$ Daniel Silver ${ }^{\mathrm{a}, *},{ }^{\infty}$ Thiago H Silva ${ }^{\mathrm{a}, \mathrm{b}}$ \\ ${ }^{a}$ University of Toronto, Toronto, Canada \\ ${ }^{b}$ Universidade Tecnologica Federal do Parana, Curitiba, Brazil \\ $\infty$ Equal contribution to this work.
}

\begin{abstract}
Why some neighbourhoods change over time but others retain their identity remains an open question. Several attempts have been made to answer this question, with a family of models emerging as a result. However, empirically evaluating neighbourhood evolution models is a challenging task, because most require information that is difficult to obtain in traditional sources. For this reason, researchers have turned to new datasets, such as census microdata, Twitter, and Yelp. In this study, we articulate a functional model of neighbourhood change and continuity, adapted from a classical functionalist model proposed by Stinchcombe in 1968. We argue this model provides a relatively simple way to capture key aspects of the complex causal structure of neighbourhood change that are implicit in much neighbourhood change research but rarely formulated explicitly. We demonstrate how to assess the proposed model empirically using largescale data from Yelp.com. Our results indicate that our approach can potentially help to understand the nature of neighbourhood change and be useful in different applications. Keywords: Neighbourhood Change, Functional Model, Yelp Data
\end{abstract}

\section{Introduction}

Why some neighbourhoods retain their identity over time but others change is among the foundational questions of social science [1]. From the early Chicago School

\footnotetext{
* Corresponding author

Email address: dan.silver@utoronto.ca ( ${ }^{\infty}$ Daniel Silver)
} 
forward, researchers have pointed to competition for valuable space among individuals and groups, with varying factors moderating the outcome, such as money, political clout, sentimental attachment, social cohesion, broader national and state institutions, and more [2]. Concepts like invasion-succession, filtering, local subcultures, tipping, and growth machines emerged to describe what is evidently a complex, non-linear, multi-dimensional process [3].

To capture this process, some scholars have produced highly complicated attempts at comprehensive models, sometimes involving dozens of interacting variables feeding back into one another [4, 5]. The overwhelming nature of such models leads others to articulate families of simpler models. Galster [3], for example, identifies multiple mechanisms by which neighbourhoods change or maintain themselves at critical thresholds, such as collective socialization, gaming, tolerance, contagion, and diminishing returns. Such families of models offer social scientists toolkits for explaining neighbourhood dynamics without pretending to provide the final complete account [6].

Empirically evaluating models of neighbourhood evolution has proven difficult, however. Since most seek to identify micro-processes driving aggregate change or stability, they require fine-grained, longitudinal, geospatial individual, household, or business data. For this reason, while a number of studies have shown that non-linear thresholds characterize neighbourhood change, "unfortunately none of these studies directly test which of the aforementioned mechanisms are primarily at work" [3]. A growing literature on neighbourhood change promises to remedy this situation [7, 8]. New large-scale and highly detailed data sources promise to improve this situation, especially those derived from digital traces of individual and organizational activity patterns and administrative sources. Accordingly, researchers have turned to tax and real estate records, census microdata, Facebook, Twitter, Foursquare, Yelp, and more [9, 10, 11, 12, 13, 14].

Working with a family of mechanistic models allows researchers to use such data to evaluate their explanatory potential in different settings. They also provide platforms for formulating additional mechanisms only implicitly accounted for in existing models. For example, existing accounts routinely refer to processes by which new groups challenge current occupants, and the latter respond in ways that either maintain the 
existing neighbourhood identity or give way under pressure [15, 16, 17]. While concepts such as "collective efficacy" point in this direction [18], this is not an intrinsic property of some communities. Rather, it is a functional relationship that only emerges in practice: when challenged incumbents exert counter-pressure against challengers. To model such a functional relationship requires a functional model of neighbourhood change and continuity. The major goal of this paper is to propose such a model and illustrate an empirical application. To this end, the main contributions of our study are:

- Formulation of a functional model of neighbourhood change and continuity, adapting the model developed in [19].

- Deriving from this model six hypotheses to test the presence and strength of complex causal processes of neighbourhood change and continuity, such as "homeostatic response," "structural reinforcement," and "equifinality."

- Illustration of an approach to measuring the model's elements from georeferenced activity data, using Yelp.com as a case study.

- Demonstration of how to empirically evaluate the proposed model. We utilize large-scale data from Yelp.com to study the performance of our proposed model.

- Demonstrating that, across six cities, neighbourhoods exhibit clear signs of functional processes. All six predictions were confirmed. This illustrates the value of functional explanation for contemporary social research and Big Data's utility for studying neighbourhood change and persistence.

The remainder of this study is organized as follows. First, we review related work on functional explanations, neighbourhood change, and the use of social media data to study cities and communities. Second, we formulate a functionalist model of neighbourhood change, and derive six testable hypotheses from this model. Third, we elaborate on how to represent this model with Yelp.com data and map specific predictions for each hypothesis based on this representation. Fourth, we test the hypotheses, initially by zooming in on four Toronto neighbourhoods, and then by generalizing across six cities: Calgary, Charlotte, Montreal, Phoenix, Pittsburgh, and Toronto. Finally, 
we conclude by discussing the significance and limits of the study as well as future directions.

\section{Related work}

\subsection{Functional explanation}

Functional explanations, broadly speaking, regard some consequences of a social arrangement or activity as crucial causes of the arrangement or activity in question [19, 20]. For example, an urban researcher who hypothesizes that gated communities' guard stations exist to maintain the social standing of their residents by excluding members of marginalized groups is proposing a functional explanation. The consequence, "maintaining social standing," is hypothesized to be the cause of the social arrangement, "guard stations."

While functional explanation has been characterized as "what any science does" [21], it has largely fallen out of favour in social science [22]. There are several common criticisms [23]. These include charges that functionalist explanations assume overarching goals shared by entire social systems, thereby denying the possibility of social conflict - functional unity; that all social entities, if they exist, must therefore serve some (possibly latent) social function, leaving the researcher free to posit functions even for random or contingent arrangements - universal functionalism; and that some functions, usually served by traditional institutions, are so important that they cannot be altered, fostering unwarranted complacency and conservatism - functional indispensability. These criticisms were widespread through the 1960s and 1970s, and were especially potent when directed against Parsonian structural-functionalism and its anthropological cousins [22]. They persist today [24], even as functional explanation is less frequently advanced, at least explicitly.

Despite these and other criticisms, functional explanation needs not to assume society's functional unity, universal functionalism, or functional indispensability. For any proposed functional explanation, one must ask: functional for whom? Conflict is, therefore, a constant possibility in a functional account [25]. Similarly, universal functionalism is not inherent to functional explanations. When properly specified, a 
functional hypothesis can be falsified: a purported function may fail to exhibit the expected set of functional relationships [26] (p. 80). For example, suppose empirical observation shows that members of marginalized groups frequently become residents of gated communities. In that case, one might conclude that the guard station does not function to exclude such groups.

Moreover, far from assuming complacent conservatism, functional accounts are crucial in much critical social theory. From Marx and Engels' claim that, under capitalism, governments exist to serve the interests of the bourgeoisie, to more recent arguments by Pierre Bourdieu that social connections and cultural attainment do so as well, critical theorists have made strong claims about how the purported functions of various social arrangements perpetuate their existence [27, 28]. For these and similar reasons, recent philosophers and theorists of social science have offered "qualified defences" of functional explanation [22, 26, 28].

The difficulty of avoiding functional explanation is evident in the fact that, despite its bad reputation, social scientists routinely use functional motifs in their accounts of social life. Jackson [22] shows their ubiquity in economic theory. In our review of the literature on neighbourhood change, we found numerous examples as well [29, 30, 31, 32, 33, 34, 35, 36, 37, 38, 39. All in all, functionalist intuition remains commonplace in social research in general and neighbourhood change research in particular.

Perhaps because of the legacy of its stigmatization, however, researchers rarely articulate such functionalist motifs as such. Nor do they formulate them in terms of a model that would allow their implications to be evaluated empirically. This is not unusual: "references to functional relations in the social scientific literature are seldom linked with evidence that these relations do exist." [26] (p. 58). In fact, we found no examples in the neighbourhood change literature where an author who utilized a functionalist motif articulated the motif in an explanatory model that would render it testable, let alone directly testing the claim. The major goal of this study is to demonstrate how this type of social research can be revived in the context of the big data revolution. To do so, we return to the classical empirical model of functional explanation formulated by Stinchcombe in 1968 and adapt that model to contemporary Yelp data to study neighbourhood change. We view this dialogue between classical mod- 
els and novel data sources as important to advancing the utility of big data for social research.

\subsection{Yelp as a source for studying neighbourhood change}

We explore data provided by Yelp.com to illustrate an empirical strategy for evaluating hypotheses generated from the functionalist model of neighbourhood change, articulated below. Yelp is one of the most popular consumer review social media, with more than 150 million unique users providing millions of reviews in several different languages [40]. Yelp has much to offer as a source of data for studying neighbourhood change. In particular, it contains information about (i) points of interest (POI) and (ii) their users. POIs are mainly businesses such as restaurants and coffee shops, but also may include public spaces such as parks or hiking trails. Each POI is classified within a hierarchical list of categories and may contain reviews provided by users containing evaluative assessments. The reviews are date stamped and correspond to a GPS location, enabling geospatial and temporal analysis. Furthermore, each user reviewer accumulates a portfolio of reviews and their locations, allowing understanding their taste profile by the number and type of places of their reviews.

Yelp and other location-based social networks (LBSNs) have provided an avalanche of data for urban and regional scholarship. Twitter has been a common source. For example, in a recent study, researchers used geo-located tweets to measure the social integration of U.S. cities based on the everyday travels of people across neighbourhoods [41]. Nevertheless, it is worth noting that Twitter data, even when georeferenced, is limited in its geographic scope since there is no guarantee that a tweet posted from a given location is about that location.

Foursquare and Yelp data provide an alternative that avoids this issue. They have been used to locate groups of users in the city by analyzing the places they visit [42], to distinguish cultural boundaries by looking at food and drink habits [13], to identify the sources of urban "buzz" [43], to identify neighbourhood boundaries through patterns of collective activities and social proximity [12, 13], and in urban planning to allocate retail stores in the city better [44]. Other studies have shown that location-based services accurately predict and monitor changes in local socioeconomic attributes [45, 9] 
and extract urban patterns [46], including the perceived quality of an area using Google StreetView data [47], and meaningful spatiotemporal insights of events such as disease outbreaks or social activities [48]. Zukin, Lindeman and Hurson [49] identify racial bias by manually coding Yelp reviews of restaurants in two gentrifying neighbourhoods —one predominantly Black and another predominantly White. Olson et al. [50] use computational text analysis techniques to "read the city" via Yelp reviews, revealing collective representations across neighbourhoods and identifying patterns of continuity and change among them.

Our use of Yelp data builds upon and extends these past studies. Specifically, we incorporate users in interaction with venues and neighbourhoods. In this context, we derive taste groups based on the intersection of user profiles derived from the sets of POIs they review. Moreover, instead of treating those elements separately, we consider their complex interactions. Integrating this mix of venue, geographic, and user information is the first of its kind to our knowledge. It not only enables us to extract richer information, but it is also crucial for studying the complex causal structures underlying neighbourhood change.

\section{A model of neighbourhood change and continuity}

\subsection{The elementary causal structure of a functional explanation}

Social theorists Hans Joas and Wolfgang Knoebl [26] observe that "functionalism is permissible only if actual processes of feedback can be shown” (p. 80). In this paper, we follow Stinchcombe [19, 20], who develops an approach to formulating models of functionalist feedback processes more rigorously, which for this reason has been held up as a key example of functionalist explanation that avoids all but its most extreme criticisms [25]. In this approach, "functional causal imagery" is indicated whenever one observes a "pattern of equifinality," that is, relatively uniform consequences arising from diverse means. For example, societies typically have inheritance rules that determine who receives goods upon the death of their owners. Those rules vary greatly, but the consequence remains uniform, suggesting a functional explanation in terms of inheritance patterns. To specify the process implied by this sort of functionalist imagery, 
Stinchcombe articulates the elementary causal structure of a complete functional explanation in terms of four core elements:

1. The consequence that tends to be maintained, which also functions indirectly as a cause of the behaviour or social arrangement to be explained. This is $\mathrm{H}$, the "Homeostatic" variable. Though H may tend to be stable empirically, its stability is maintained against pressures to change it, such as in the case of body temperature.

2. The social arrangement or behaviour that impacts $H$, the explanandum. This is $\mathrm{S}$, the "Structure." In a functional model, $\mathrm{S}$ tends to maintain H. For example, sweat glands tend to maintain body temperature.

3. Tensions that tend to upset $\mathrm{H}$, unless $\mathrm{S}$ maintains it. This is $\mathrm{T}$, the "tension" variable. If physical activity or air temperature did not alter body temperature, there would likely be no structure to counteract the tensions they create.

4. Processes that reinforce or select for the S's that maintain $\mathrm{H}$. When $\mathrm{H}$ is threatened or pressured, these forces increase the activity of $\mathrm{S}$ when $\mathrm{T}$ is higher and decrease when $\mathrm{H}$ is maintained. For example, sweat glands generate more sweat (S) when body temperature $(\mathrm{H})$ is not maintained at normal levels due to a certain phenomenon (T). Since this structure helps to maintain $\mathrm{H}$ in equilibrium, it will tend to be selected or reinforced.

Combining these elements yields the diagram depicted in Figure 1 (left) describing the core causal structure of a functional explanation. To illustrate this diagram, Stinchcombe uses the example of magical rituals to reduce anxiety around sea fishing [19](p. 136). In that example, $\mathrm{H}$ is anxiety, $\mathrm{T}$ is uncertainty in fishing, and $\mathrm{S}$ is magical activity. As T (uncertainty) increases, so does H (anxiety). However, S (magical activity) compensates and reduces anxiety to tolerable levels. This has the effect of reducing variation in $\mathrm{H}$, maintaining relatively stable anxiety levels over time. Moreover, $\mathrm{S}$ (magical activity) becomes selected out and reinforced in proportion to its effectiveness in maintaining tolerable anxiety levels; were other more effective structures (e.g. improved ship design or weather forecasting) to emerge, they would tend to be reinforced instead. There is, therefore, a close linkage between functional explanations and 
evolutionary theory [51].
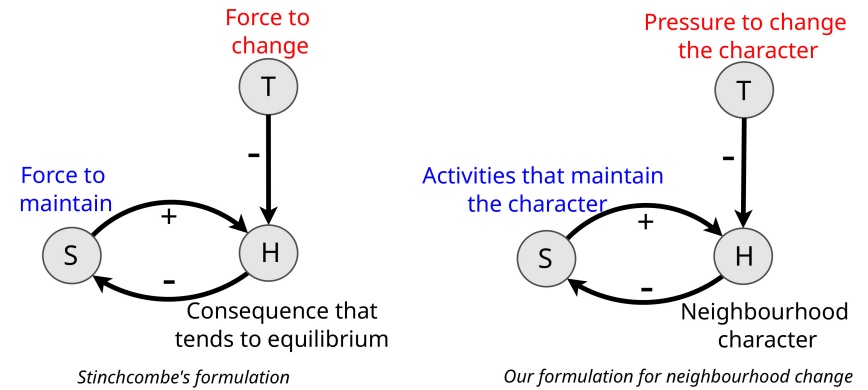

Figure 1: Core causal structure of a functional explanation: Stinchcombe's articulation (left), our proposition in the context of neighbourhood change (right).

Stinchcombe and others working with this model [25] have extended it in various ways. For example, Stinchcombe decomposed the Structure into multiple groups with differential interests and power, such as aristocrats, bourgeoisie, and proletariat. Depending on their power, the model can be used to make predictions about the probability of maintaining a particular form of government, such as parliamentary democracy. Arditi [25] added a loop from $\mathrm{H}$ to $\mathrm{T}$ to capture the possibility of structural contradictions, where efforts to sustain a homeostatic equilibrium only provoke greater tensions. While we do not adapt these particular extensions to our neighbourhood change model, they show the model is not fixed but open to adaptation. In our case, we decompose the groups in $\mathrm{T}$, which allows us to compare them to those in $\mathrm{S}$ to identify situations that should hypothetically generate greater or less pressure to change, as outlined below.

\subsection{Functional explanation of neighbourhood change and continuity}

Stinchcombe's diagram in Figure 1 (left) may be intuitively mapped onto familiar neighbourhood dynamics, as in Figure 1 (right). For example, we may consider $\mathrm{H}$ as neighbourhood character, style, or scene [52] (such as distinctive shops, restaurants, venues, or groups), $\mathrm{T}$ as pressures to change that character (from, for example, new groups with divergent tastes), and $\mathrm{S}$ as activities that maintain that character (such as Business Improvement Association sponsored festivals, political advocacy, or increased participation in venues and activities distinctive to that scene). 
Based on this simple representation, we can formulate an initial set of hypotheses regarding the presence and strength of 1) a functional relationship and 2) a homeostatic response. We first present these and our other hypotheses in a highly abstract fashion before operationalizing them more concretely below.

\section{H1-H3: Functional relationship hypotheses.}

- H1: Equifinality. Neighbourhoods are expected to be more similar to themselves over time than to other neighbourhoods, i.e., relative stability and self-similarity in $\mathrm{H}$ are expected in general.

- Widespread self-similarity over time across many neighbourhoods is an indication of equifinality: diverse conditions generate a relatively uniform outcome. This would suggest a functional explanation of neighbourhood character.

- H2: Structural response. Local increases in T should be followed by increases in $\mathrm{S}$.

- If neighbourhoods function to maintain their existing character, as pressures to change grow, this should be followed by increases in activities that correspond to that character.

- H3: Significant response. Increases in S should be proportional to the strength of $\mathrm{T}$ to which they are subjected, i.e., the increase should also be substantial.

- If neighbourhoods function to maintain their existing character, the structural response should not only follow rising Tension (H2); it should be larger when the Tension is larger.

\section{H4: Homeostatic hypothesis.}

- H4: Homeostatic response. If $\mathrm{H} 2$ and $\mathrm{H} 3$ hold, $\mathrm{H}$ should exhibit reduced variability over time. By contrast, if $\mathrm{H} 2$ and $\mathrm{H} 3$ do not hold, $\mathrm{H}$ should exhibit greater change over time. 
- If $\mathrm{H} 1$ tests whether a neighbourhood tends to maintain itself, $\mathrm{H} 4$ determines if this self-similarity is more likely to occur where the functional Tensionresponse relationships $(\mathrm{H} 2-\mathrm{H} 3)$ obtain.

Extending these implications of the basic functionalist model, we formulate two additional propositions by decomposing the Structure and Tension variables. This decomposition allows us to articulate hypotheses about 1) the presence of a reinforcement process and 2) the strength of the pressure to change generated. Figure2 2 illustrates the intuition behind these hypotheses.

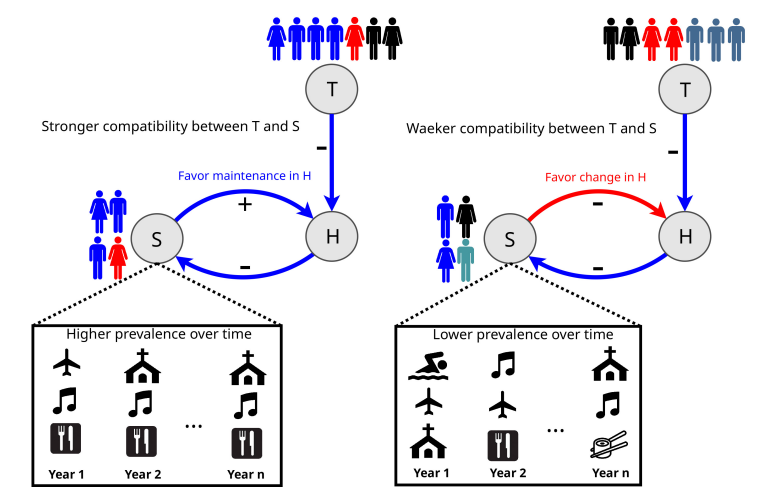

Figure 2: Illustration of the logic behind hypotheses H5 and H6.

Figure 2 illustrates the logic behind H5, the structure reinforcement hypothesis. The boxes unpacking $\mathrm{S}$ show the categories defining the structure activities over time. On the left, where a functional explanation obtains (i.e. where $\mathrm{H} 2$ and $\mathrm{H} 3$ hold, "favouring maintenance"), the activities supporting the existing neighbourhood character are retained through time. This suggests a selection process reinforcing the structure. By contrast, on the right, where the functional response is not present (i.e. where $\mathrm{H} 2$ and $\mathrm{H} 3$ do not hold, "favouring change"), the structure activities are not reinforced, and their prevalence is lower over time.

\section{H5: Structure reinforcement hypothesis.}

- H5: Structure reinforcement. S's that effectively respond to T by maintaining H should be reinforced over time. 
- If neighbourhoods function to maintain their existing character (i.e. H2 and H3 obtain, "favouring maintenance"), then the activities that sustain the neighbourhood's existing structure (those that characterize S) should tend to be selected or reinforced.

Figure 2 also illustrates the logic of H6, the Structure-Tension compatibility hypothesis. The people in $\mathrm{T}$ and $\mathrm{S}$ have different colours, indicating their shared taste profiles (blues are a group with a similar taste profile, reds with a different profile, etc). On the left, the new groups visiting a neighbourhood (those in $\mathrm{T}$ ) predominantly share taste profiles similar to those characterizing the local structure activities (those in $\mathrm{S}$ ). In this situation, the pressure from visitors with divergent tastes is more likely to be overwhelmed by those with tastes more compatible with the existing structure activities, making the structural response more effective. Therefore, this pattern is more likely where $\mathrm{H} 2$ and $\mathrm{H} 3$ hold ("favouring maintenance"). By contrast, in the right diagram, new visitors predominantly have divergent tastes. In this situation, the Tension should be stronger, and the structure activities should be less likely to successfully exert counter-pressure. Here, change should be favoured.

\section{H6: Structure-Tension compatibility hypothesis.}

- H6: Structure-Tension compatibility. Areas where H2 and H3 hold should present stronger compatibility between $\mathrm{S}$ and T; by contrast, where $\mathrm{H} 2$ and $\mathrm{H} 3$ do not hold, $\mathrm{T}$ and $\mathrm{S}$ should diverge.

- If neighbourhoods function to maintain their existing character, when new visitors are more compatible with the forces that successfully realize this outcome, those forces should more effectively counteract any pressure to change.

\subsection{Representing elements of the neighbourhood change's functional model using Yelp}

We use Yelp data to represent the key variables in the functionalist model.

- To measure $\mathrm{H}$, the existing neighbourhood character, we use the distribution of types of business, i.e., their types and quantities. 
- To capture Tension (T), the pressure to change the local character, we consider the distribution of distinct groups' visits in a specific area. Groups are defined according to the taste profiles revealed in the types of establishments they review. In a given area, if in consecutive years the distribution of groups changes, we consider this an indication of increasing Tension: new groups have appeared with tastes that diverge from those that have previously predominated in the area.

- To measure Structure (S), activities that maintain the local character, we consider recurrent users' visits to venues in a neighbourhood. In this way, we measure S as popular types of venues that implicitly represent existing groups' preferences through activities they like to perform in a certain area. The Structure may be composed of multiple groups; they need not be defined by one particular type of user.

Below we specify this mapping of model to data more formally. This allows us to formulate empirical predictions for evaluating H1-H6.

- Prediction 1: Equifinality. Areas are expected to be more similar to themselves over time than other areas, according to their distribution of business categories;

- Prediction 2: Structural response. An increase in groups with different preferences (Tension T) in an area should be followed by an increase of the use of popular types of venues in an area by recurring users (Structure S);

- Prediction 3: Significant response. Increases in both $\mathrm{S}$ and $\mathrm{T}$ should be above recent trends;

- Prediction 4: Homeostatic response. Areas that "favour maintenance" - in which $\mathrm{H} 2$ and $\mathrm{H} 3$ tend to hold - will change less over time in terms of the distribution of business categories, compared to areas that "favour change," where H1 and $\mathrm{H} 2$ do not tend to hold;

- Prediction 5: Structure reinforcement. The intersection set of business categories composing the Structure of the same tract over time will tend to be pro- 
portionally higher in areas in which $\mathrm{H} 1$ and $\mathrm{H} 2$ tend to hold than in areas where $\mathrm{H} 1$ and $\mathrm{H} 2$ tend not to obtain;

- Prediction 6: Structure-Tension compatibility. Areas where H1 and H2 tend to hold will exhibit a stronger similarity between categories composing $\mathrm{S}$ and the groups predominant in $\mathrm{T}$.

\section{Data and methods}

This section describes the data used and the methodological procedures applied to them, summarized by Figure 3 .

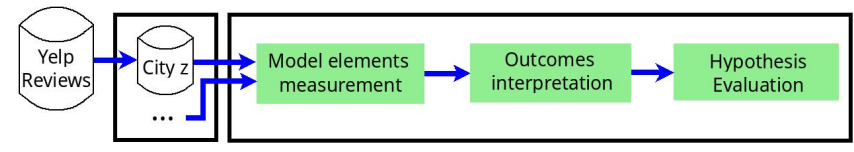

Figure 3: Overview of the methodological steps.

\subsection{Yelp data}

We use an official dataset provided by Yelp [53]. This dataset represents a subset of Yelp businesses, reviews, and user data from 2008 to 2018. This study considers the three most popular cities in this dataset in Canada (Calgary/Alberta, Montreal/Quebec, and Toronto/Ontario) and in the United States (Charlotte/North Carolina, Pittsburgh/Pennsylvania, and Phoenix/Arizona). For each city, we have the count of establishments, information about each POI's category (e.g. "hair salon" or "coffee shop") and all of its reviews. Each review is also indexed to the reviewer who wrote it. Finally, we have a Business ID, a User ID, and a location (lat/lon) associated with each review. Table 1 summarizes the number of businesses, reviews and users for all cities considered in this study.

To evaluate the dataset's coverage, we compared business counts to national business registries, such as the County Business Patterns $s^{1}$ or Canadian Business Count $\mathrm{s}^{2}$.

\footnotetext{
${ }^{1} \mathrm{https} / / /$ www.census.gov/programs-surveys/cbp.html.

${ }^{2} \mathrm{https}: / /$ www.statcan.gc.ca.
} 
Table 1: Overall statistics of the studied dataset.

\begin{tabular}{llll} 
& \# Businesses & \# Reviews & \# Users \\
\hline Calgary & 7,736 & 97,650 & 34,645 \\
Charlotte & 9,509 & 308,793 & 132,238 \\
Montreal & 6,602 & 155,966 & 58,660 \\
Phoenix & 18,766 & 730,244 & 369,288 \\
Pittsburgh & 7,017 & 224,423 & 93,168 \\
Toronto & 18,906 & 525,435 & 169,516
\end{tabular}

Following [9], we considered the restaurant industry, a category with significant coverage by Yelp and readily comparable to government business registry categorizations. This evaluation indicated that data in general before 2011 are less consistent with the census, likely because the Yelp platform was less developed then. The evaluation for Toronto is illustrated in Appendix B For this reason, the remainder of this study considers only data from 2011 to 2018 . In the rest of the study, we also only consider areas (census tracts) with at least 50 unique businesses to avoid predominantly residential areas.

\subsection{Measuring the model's elements}

For the sake of simplicity, we explain our methodology for a specific city $z$. However, we repeat the same steps for all cities.

\subsubsection{Structure}

The Structure is extracted in four main steps. 1-representation of areas by number of reviews in their business category. For each city we identify a set of unique venue categories $C=\left\{c_{1}, c_{2}, \ldots, c_{j}\right\}$, where $j$ is the number of unique categories observed in a particular city under evaluation. Then, we represent each area, i.e., tracts, of a given city by the number of reviews $(r)$ performed by users in venues of each category $\left(c_{i} \in C\right)$ in a certain area $A_{i}$ during year $y: A_{i}^{y}=\left\{r_{c_{1}}^{A_{i}, y}, \ldots, r_{c_{x}}^{A_{i}, y}\right\}$. With that, $A_{i} \in \mathcal{A}=\left\{A_{1}^{y_{1}}, \ldots, A_{1}^{y_{n}}, \ldots A_{x}^{y_{1}}, \ldots, A_{x}^{y_{n}}\right\}$, where $n$ represents the number of years covered by the dataset, and $x$ the number of tracts available on a certain city. When studying data from a particular year $y$, we only consider the activity of a certain user in the area $A_{i}^{y}$ if he/she was observed in the same area in the past (at least $y-1$ ). 
We disregard this condition only in the first year studied. Also, we take into account only one visit per user in the same venue per year, which permits a more representative characterization of users' preferences. All users considered are registered in $\Omega$. The rest of the analysis considers a normalized version $([0,1])$ of each vector $A_{i}^{y}$.

2-Identification of core categories. For a particular area and point in time $A_{i}^{y}$, we separate the activity from two previous years $A_{i}^{y-1}$ and $A_{i}^{y-2}$. Then, we sort each of them in descending order in terms of reviews per categories. Going from higher to lower values, we gather all categories and their respective number of reviews in $W_{i}$ until it reaches $p \%$ of all reviews in the vector under consideration, i.e., $A_{i}^{y-1}$ or $A_{i}^{y-2}$. Thus, $W_{i}^{y-1} \subseteq A_{i}^{y-1}$ and $W_{i}^{y-2} \subseteq A_{i}^{y-2}$. In our experiments, we evaluate $p \in\{30,40,50,60\}$. For each city, we consider the value of $p$ that provides the best overall performance (see Section 4.4.1.

3- Structure value calculation. We find the set of categories $C_{\text {match }} \subseteq C$ representing categories that appeared in both $W_{i}^{y-1}$ and $W_{i}^{y-2}$. Then, we sum the reviews counts in $A_{i}^{y}$ for the categories in $C_{\text {match }}$, and this value is the Structure value $s \in S_{A_{i}}=\left\{s_{A_{i}}^{y_{1}}, s_{A_{i}}^{y_{2}}, \ldots, s_{A_{i}}^{y_{n}}\right\}$. If $C_{\text {match }}=\emptyset$, then $C_{\text {match }}$ is considered to be the categories in $W_{i}^{y-1}$. Thus, the Structure represents users who are not new to the focal area and performed a review consistent with the most popular business categories in that area.

Finally, 4- Peaks identification. We identify significant peaks in the Structure time series, i.e., peaks that stand out from a recent trend. It is important to identify significant peaks because, in the context of our model, they represent periods where forces were being applied more intensely. A peak is spotted if a certain Structure value $s_{A_{i}}^{y}>\mu+\sigma$, where $\mu$ and $\sigma$ represents the mean and standard deviation of the set $\left\{s_{A_{i}}^{y-1}, s_{A_{i}}^{y-2}\right\}$, which represents Structure values of the two most recent years in the past. Note that the first peak identification is only possible from 2013. Thus, for each tract $i$ we have a vector representing Structure peaks: $S P_{i}=\left\{q^{y_{2013}}, \ldots, q^{y_{2018}}\right\}$, where $q$ is 0 (no peak) or 1 (peak). 


\subsubsection{Tension}

We perform five steps to extract the Tension. 1- User representation by categories of business visited. We describe each user, not in $\Omega$, by the names of categories of places they performed a review. In this way, users can be seen as "documents," and the category names, which can be repeated, are words in these documents. This is one example: $d_{1}=\{$ Supermarket, Coffee Shop, Book Shop, Coffee Shop $\}$, where $d_{1} \in D$ represents a document describing user1. By not considering users in $\Omega$ we maximize the chances of working with new users to a certain area.

2- Profiles extraction and representation of users in this space. We apply standard text pre-processing steps on all documents $D$ representing users, removing numbers, special characters and blank spaces, and use these cleaned documents to identify latent topics. For this task, we use Latent Dirichlet Allocation (LDA) [54], a technique helpful to express documents in a set of topics, finding words that describe the subject of a document. The intuition behind LDA is that each document can have several topics, and each topic is a distribution of probabilities for a word in the vocabulary. In our context, topics can be interpreted as user profiles; thus, users can be associated with different profiles, as in real life. We explore UMass topic coherence measure [55], which considers the original corpus used to train the topic models, rather than an external corpus as in other coherence metrics. We use the coherence scores to evaluate different scenarios: 1 to 30 topics. We look at this distribution of coherence scores to identify a suggested number of topics, i.e., the highest score, and choose this value and its immediate before and after neighbours' values to proceed with our analysis. After identifying the topics, we map each document (which represents users) on the space defined by those topics: $u=\left\{x_{1}, x_{2}, \ldots, x_{n}\right\}$, where $u \in U$ is a feature vector representing a particular document (user), and $x_{i}$ represents the probability that this user is associated with a certain topic (profile) among $n$ topics considered.

3- Define groups by users' profiles. We find groups of users $G=\left\{g_{1}, \ldots, g_{k}\right\}$ in the space represented by the feature vector $U$. For that, we use the $k$-means algorithm, where $k$ is identified according to the silhouette heuristic (evaluating $k$ from 2 to 15 ).

4- Representation of tracts by distribution of Groups' visits. Each tract of a certain 
city and year $y, V_{i}^{y}$, is represented by the number of unique visits of each group $\left(f_{g_{i}}\right)$ : $V_{i}^{y}=\left\{f_{g_{1}}^{V_{i, y}}, \ldots, f_{g_{k}}^{V_{i, y}}\right\}$, in this way, $V_{i} \in \mathcal{V}=\left\{V_{1}^{y_{1}}, \ldots, V_{1}^{y_{n}}, \ldots V_{x}^{y_{1}}, \ldots, V_{x}^{y_{n}}\right\}$, for $n$ years covered by our dataset, and $x$ tracts. We use this representation to calculate the cosine distance between all pairs of consecutive years of the same area: $t=\operatorname{cosine}\left(V_{i}^{y^{\prime}}, V_{i}^{y+1^{\prime}}\right)$, where cosine is the cosine distance, and each vector $V^{\prime}$ is the normalized $([0,1])$ version of $V$. In this way, if groups visiting a certain area do not change significantly from year $y$ to $y+1, t$ will be close to zero, whereas, if they are completely different, the value will be 1 . In the end, we have a vector $T_{V_{i}}=\left\{t_{V_{i}}^{y_{1}}, t_{V_{i}}^{y_{2}}, \ldots t_{V_{i}}^{y_{n}}\right\}$, representing a time series of Tension values for a particular tract.

5- Peaks identification. We identify peaks in the Tension time series for each tract. A peak is spotted if a certain Tension value $t_{V_{i}}^{y}>\mu+\sigma$, where $\mu$ and $\sigma$ represents the mean and standard deviation, respectively, of the set $\left\{t_{V_{i}}^{y-1}, t_{V_{i}}^{y-2}\right\}$, which represents Tension values of the two most recent years in the past. Note that the first peak identification is only possible from 2014 since the first value of Tension is obtained from 2012. Thus, for each tract $i$ we have a vector representing Tension peaks: $T P_{i}=\left\{q^{y_{2014}}, \ldots, q^{y_{2018}}\right\}$, where $q$ is 0 (no peak) or 1 (peak).

\subsubsection{Homeostatic}

In possession of the set of unique category types $C$ observed for a certain city, we represent each tract $E_{i}^{y}$ of this city by the number of business $b$ for each category $c_{i} \in C$ during year $y: E_{i}^{y}=\left\{b_{c_{1}}^{E_{i}, y}, \ldots, b_{c_{j}}^{E_{i}, y}\right\}$. A certain business is first accounted in year $y$ representing its first review in our dataset. Every other year onward, $y+1, y+2, \ldots$, we check if this business has any review on this particular year or in the future to keep being accounted, otherwise, it is disregarded. Thus, $E_{i} \in \mathcal{E}=\left\{E_{1}^{y_{1}}, \ldots E_{1}^{y_{n}}, \ldots E_{x}^{y_{1}}, \ldots E_{x}^{y_{n}}\right\}$, being $n$ the number of years studied, and $x$ the number of tracts in a certain city. 


\subsection{Hypothesis evaluation}

\subsubsection{H1 - Equifinality}

To evaluate this hypothesis, we compute the pairwise cosine distances between normalized ([0,1]) observations of $\mathcal{E}$, obtaining $\mathcal{E}^{\prime}$. After that, for every element $E_{i} \in$ $\mathcal{E}^{\prime}$ we find the seven most similar ones (not including itself) in $\mathcal{E}^{\prime}$. Then we compute equifinality $y_{i}$, representing the percentage of closest elements from the same tract in different years.

\subsection{2. $\mathrm{H} 2$ and $\mathrm{H} 3$ - Structural and significant response}

If a functional relationship exists, Tension increases should be followed by an increase in the Structure (H2), and these increases should be significant, i.e., above recent trends (H3). Since it does not make sense to evaluate $\mathrm{H} 2$ separately from $\mathrm{H} 3$, we evaluate them together. To do so, a significant Tension peak spotted in a certain tract during year $y$ is expected to be accompanied by a significant Structure peak in the same tract during year $y$ or $y+1$ (as there is no value after 2018, it is disregarded in this analysis). When this happens, we classify this phenomenon as hit, otherwise, miss. Hits, in other words, are areas where $\mathrm{H} 2$ and $\mathrm{H} 3$ is observed. According to the logic of functional explanations, we hits and misses to label areas as "Favour Maintenance" (FM) and "Favour Change" (FC).

To determine how to apply the labels FM and FC, we adopt the following procedures. If a certain tract has more or only hit, we label it as Favour Maintenance (FM), whereas, if it has more or only miss, we label it as Favour Change (FC). In case of a tie between miss and hit, we divide the period studied in two and analyze the part that reflects the most recent years. Let $Y=\left\{y_{1}, y_{2}, \ldots, y_{n}\right\}$ represent the set of years, thus, we consider the subset $\left\{y_{n / 2}, \ldots, y_{n}\right\} \in Y$ to count misses or hits if $n / 2$ is even; if this quantity is odd the subset starts at $(n / 2)+1$. In this way, we avoid ties, and we measure the tendency, FM or FC, observed for a certain tract.

Thus, FM tracts represent areas where $\mathrm{H} 2$ and $\mathrm{H} 3$ tend to hold. On the other hand, FC tracts represent tracts where those hypotheses tend not to obtain. 


\subsubsection{H4 - Homeostatic response}

To study our hypothesis $\mathrm{H} 4$, we first separate from $\mathcal{E}$, all tracts that belong to FC $\left(\mathcal{E}_{F C} \subseteq \mathcal{E}\right)$ and FM $\left(\mathcal{E}_{F M} \subseteq \mathcal{E}\right)$. Then, we calculate tracts self-dissimilarity over the years, following a chronological order. Thus, for a tract $E_{i}$ we calculate $\operatorname{cosine}\left(E_{i}^{y}, E_{i}^{y+1}\right)$, for $y \in\{2011,2012, \ldots, 2017\}$. This process is repeated for all tracts. We use these distributions of self-dissimilarity values for each class to study this phenomenon.

\subsubsection{H5 - Structure reinforcement}

In this analysis, we compute the intersection $I_{W_{i}}^{y, \pi}$ of each $W_{i}^{y}$ with all its representation in the future $W_{i}^{\pi}$, where $\pi \in\{y+1, \ldots, n\}$ for $n$ considered years. As an example, for $W_{i}^{2016}$ we compute $I_{W_{i}}^{2016,2017}$ and $I_{W_{i}}^{2016,2018}$. Then we compute the percentage of values represented by each intersection obtained, i.e., we compute $\frac{\left|I_{i i}^{y, \pi}\right|}{|\Phi|}$, where $\Phi$ represent a set from the merge of $W_{i}^{y}$ and $W_{i}^{\pi}$. If $\Phi$ has size one and contains the element "Restaurants", we disregard the percentage calculated. This is because the repetition of a Structure that only has Restaurants does not represent a meaningful reinforcement. Almost all tracts have this category in the Structure, i.e., support is one or close to one in all cities. Next, we separate all percentages from tracts of FM and FC.

To complement this analysis, we also compute frequent sets of business categories in the Structure of different tracts. This helps to understand what specific categories tend to be reinforced among a set of tracts, and informs the concrete illustrations we provide below. Let $\mathcal{W}_{i}=\left\{W_{i}^{y=1}, \ldots, W_{i}^{y=n}\right\}$ represent all sets of categories of the Structures of tract $i$ in all years. We group all Structure categories of $\mathcal{W}_{i}$, thus resulting in a vector $W_{i}^{\prime}$ containing all categories observed in $\mathcal{W}_{i}$, where repetition is allowed. We perform this step for all tracts. Then, we separate $F M$ and $F C$ tracts to apply the Apriori Algorithm [56] with support equals 50\%. For example, considering the $F M$ tracts, we obtain $\mathcal{W}_{F M}^{\prime}=\left\{W_{1}^{\prime}, \ldots, W_{n}^{\prime}\right\}$, where $n$ represents the number of tracts classified as $F M$. We concentrate the analysis in the sets of size one, representing categories that appeared at least $50 \%$ of the time in all Structure values. 


\subsubsection{H6 - Structure-Tension compatibility}

Here we present the steps to check the similarity between Structure categories with the groups visiting a certain area. For that, we need: i) the highest topic probability for each group $G T=\left\{l_{1}, \ldots, l_{k}\right\}$, where $k$ represents the number of groups, and $l_{j}$ represents a specific topic (profile) with the highest probability for group $g_{j} \in G$ (we compute this probability by aggregating each feature vector $u_{i}$, recall from Section 4.2 .2 that this represents the probabilities of a particular user being associated to each topic in the feature space); ii) $W_{i}^{y}$ containing all of a given Structure's categories for a certain tract $i$ in a specific year $y$; and iii) the distribution probability $\Psi$ for each word (category) in the topic - recall that each topic contains all words, but typically with different probabilities.

Then, for each FM tract $i$ in a certain year $y$, we sum in $q$ the probabilities for the words in $W_{i}^{y}$ from $\Psi_{l}^{y}$ for a certain topic $l \in G T$. In the end, we have one $q$ value for each group in a given tract in time: $Q_{i}=\left\{q_{1}, \ldots, q_{k}\right\}$. Next, we normalize $Q_{i}$ by its total sum; thus, $Q_{i}^{\prime}$ sums 1 . Then Alignment $t_{i}$ represents the average value of two values of $Q^{\prime}$ corresponding to the two most prominent/influential groups in the Tension in tract $i$ in a particular year.

\subsection{Interpretation of outcomes from the model}

\subsubsection{Quantifying overall success}

We also use the hit and miss concept to compute the overall success of a particular functionalist model for the phenomenon observed. For that, for a certain tract $i$ we calculate $\delta_{i}=$ hits $_{i} /\left(\right.$ hits $_{i}+$ misses $\left._{i}\right)$, where hits $s_{i}$ and misses $_{i}$ represent the number of hits and misses observed for tract $i$. In the end, we calculate the average of $\delta$ for all tracts in the studied city representing the measure score $=\frac{1}{x} \sum_{i=1}^{x} \delta_{i}$, with $x$ representing the number of tracts in a certain city. A certain tract is considered in this calculation only if hits $s_{i}+$ misses $_{i}$ is bigger than zero. The score is used to select the combination of threshold $p$, of the Structure calculation, and number of topics, in the range considered for the Tension calculation, that provides the highest value. 


\section{Illustrative cases from Toronto}

Before evaluating the hypotheses directly, we illustrate them and the measurements underlying them with the case of Toronto to build a stronger intuition of the processes we are measuring. In effect, testing the propositions asks how well these local observations generalize.

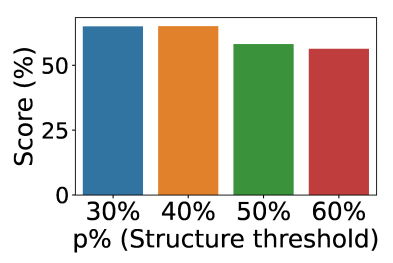

(a) 6-topics

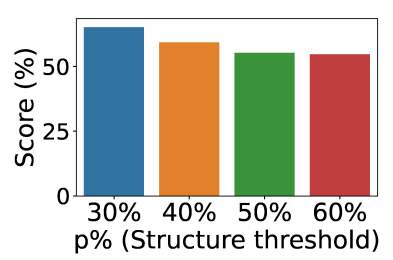

(b) 7-topics

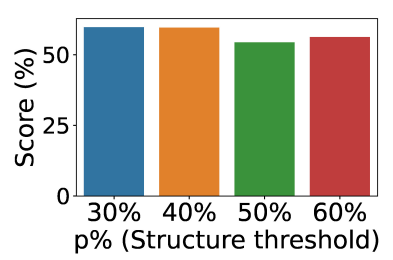

(c) 8-topics

Figure 4: Score (in percentage) for all values of $p$ under consideration in the structure calculation for Toronto. Values are shown for the range of topics suggested as good candidates.

Figure 4 shows the score (in percentage) for all values of $p$ under consideration in the Structure calculation for Toronto. Values are shown for the range of topics $(6,7$, and 8) suggested as good candidates, as explained in Section 4.2.2. Note that the best threshold for this city is $40 \%$ with six topics; thus, this configuration is explored in the rest of the analysis regarding this city. Note also that the results do not vary drastically between the number of topics and different $p$ structure thresholds. These results are similar to what is observed for the other cities.

Using the score, we calculate Structure and Tension forces, according to the procedures described in Sections 4.2.1 and 4.2.2 Figure 5(a) illustrates the resulting measurements for tract 0090, located in downtown Toronto. In this figure, the green area represents the window explored to calculate the mean $(\mu)$ and standard deviation $(\sigma)$ in the year 2016 for both forces. These values are necessary to identify significant peaks. According to our methodology, the considerable increase in the Tension force in 2016 was identified as a significant peak (see Figure 5(b) . Note that a peak is not identified for the Structure force in that particular year; however, a peak was identified for the Structure force in 2017. 


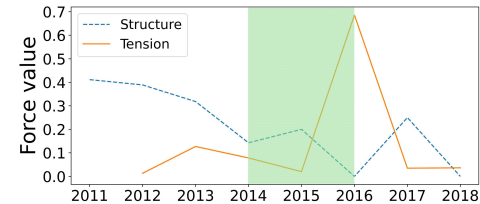

(a) Measured forces

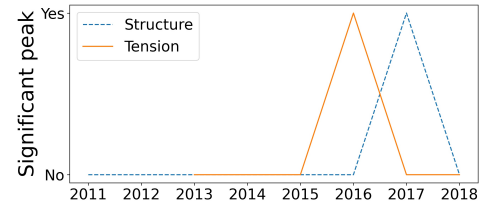

(b) Peaks identification

Figure 5: Illustration of tension and structure for tract 0090 in Toronto (a) and its identified peaks (b).

The mapping of reactions from the Structure to Tension is illustrated by Figure 6. A hit is coloured in blue and a miss in red for all the tracts studied in Toronto (columns). The gray area represents the period where a valid hit or miss can occur. In this figure, the yellow column represents tract 0090, the same one shown in Figure 5(b). In Toronto, the score $_{\text {Toronto }}=0.65$. Robustness checks for this result - and also for other cities - confirm that the phenomenon measured is not random (see Appendix A. The preponderance of blue hits in Figure 6 is an indication that $\mathrm{H} 2$ and $\mathrm{H} 3$, structural and significant response, tends to hold in Toronto. Overall, the city tends to favour maintenance (FM)

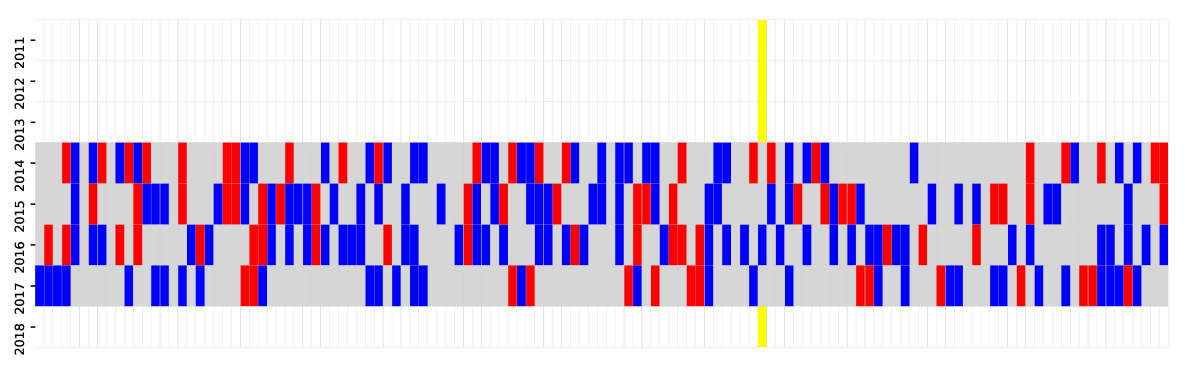

Figure 6: Mapping of hit (blue) and miss (red) for Toronto's tracts. Tract's names omitted to favour legibility. [Better in colour]

These measurements provide the backbone of our analysis. To provide concrete examples revealed by the dynamic relationship between Structure and Tension, we selected four distinct regions in Toronto. Each of these regions contains multiple census tracts. 
1. The Ossington are ${ }^{3}$ It is located in the West End of the city, home to a thriving art and nightlife scene and strong Portuguese and Vietnamese ethnic communities. The neighbourhood recently received prominent news coverage when a restaurant was accused of cultural appropriation and pressured by the community to change [57] - an intuitive example of a strong structural response that our model should be able to identify.

2. Parkdale A "bohemian" area, also in the West End, with a strong community presence, local arts groups, and diverse ethnic communities. In this neighbourhood, public fights over its identity have been pronounced over the past decade, and local community groups have organized to maintain the local scene, for instance, by prohibiting backyard and rooftop patios or campaigning against an effort to brand the area as "Vegandale" [58]. This is another case where we should observe a strong structural response.

3. Yorkville 5 This is a busy area in the central city, marked by distinctive highend fashion and restaurant scenes, as well as upscale shops and condominiums. While it has a visitor profile distinct from Ossington and Parkdale, the strongly distinctive character of its scene suggests that it is likely to favour maintenance - establishments that fail to conform to the more glamorous high-end character of the area are unlikely to succeed.

4. Danforth Village ${ }^{6}$ Located in the far East End. This is a more transitional area whose identity has been in flux and which is less clearly defined by a distinctive set of groups who actively seek to maintain its character. We would therefore expect our model to identify this neighbourhood as favouring change.

An indication that a functional process could be happening is the tendency of equilibrium of the Homeostatic variable $\mathrm{H}$ [19]. H1, in our context, measures if tracts are more similar to themselves over time than other areas (Equifinality), according to

\footnotetext{
${ }^{3}$ https://en.wikipedia.org/wiki/Ossington_Avenue.

${ }^{4}$ https://en.wikipedia.org/wiki/Parkdale,_Toronto\#Character.

${ }^{5}$ https://en.wikipedia.org/wiki/Yorkville,_Toronto.

${ }^{6}$ https://en.wikipedia.org/wiki/East_Danforth.
} 
their distribution of business categories. Recall that an equifinality value of 100 indicates that a given area in a given year was more similar with itself in different years than any other area at any point in time. The median value of equifinality for all illustrative areas of Toronto is 100 ; thus, according to H1, they are all good candidates to be explained by a functional process.

Figure 7 shows the mapping of hit (blue) and miss(red) for tracts representing each area. In Ossington and Parkdale, the number of hits is very high, and in all tracts within these neighbourhoods, we have a tendency towards structural and significant response to Tensions $(\mathrm{H} 2 \& \mathrm{H} 3)$ that favours maintenance. This is in line with expectations. Yorkville also favours maintenance, in line with our expectations. Danforth Village, by contrast, is characterized by "misses," i.e. it favours change. Taken together, these examples illustrate the face validity of our measurements.

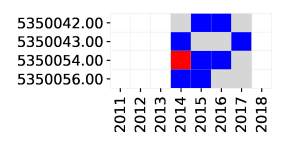

(a) Ossington

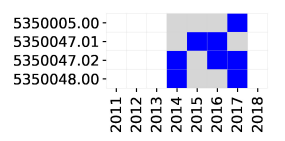

(b) Parkdale

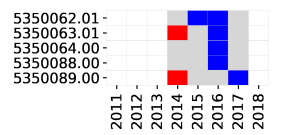

(c) Yorkville

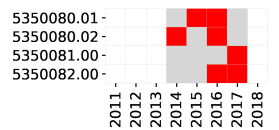

(d) Danforth Village

Figure 7: Mapping of hit (blue) and miss (red) for tracts representing four distinct areas. [Better in colour]

If Figure 7 shows which neighbourhoods are more likely to maintain or change their Structures, Figure 8(a) adds that maintenance or change tends to depend on the functional responses. The tracts in these four areas that favour maintenance - where increases in Tensions are followed by increases in Structure activity - in fact are more likely to remain more similar to themselves over time compared to tracts where the Structure fails to respond to rising Tensions. This indicates support for H4, the homeostatic response, in these neighbourhoods.

What types of activities characterize these structures, and are structures that function effectively to maintain the neighbourhood character reinforced? As defined in Section 4.3.4, we characterize $\mathrm{S}$ by the most frequent sets of size one (representing single categories). Note that diverse combinations of those sets are possible, and they do not necessarily appear together simultaneously in all tracts. Instead of discussing those combinations, we highlight frequent categories. In Ossington, the fre- 


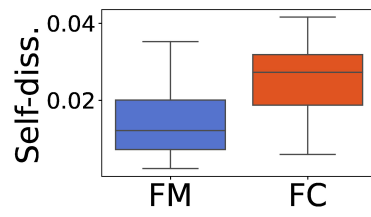

(a) Self-dissimilarity

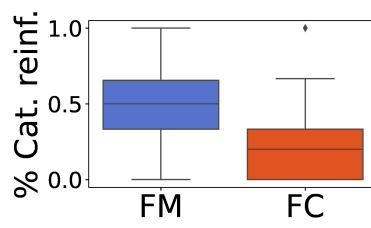

(b) Category reinforc.

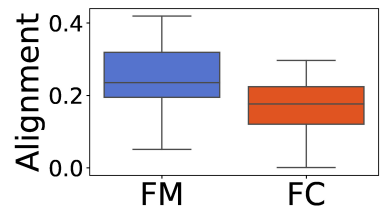

(c) Alignment

Figure 8: Results for hypothesis H4, H5, and H6 for illustrative areas of Toronto.

quent categories are "Bars, Food, Italian, Nightlife, Restaurants," in Parkdale "Bars, Food, Nightlife, Restaurants," in Yorkville "Bars, Food, Japanese, Nightlife, Restaurants, Shopping," and in Danforth Village "Bars, Food, Japanese, Nightlife, Restaurants, Shopping, SushiBars." While the frequent parts of the Structure in Yorkville and Danforth Village are considerably similar, they produce a different outcome according to our model. As shown in Figure 8(b), the structure activities are more likely to be reinforced in areas that favour maintenance. Thus in Yorkville, S in 2018 is composed of activities largely similar to those in 2011. However, where change is favoured (as in Danforth Village), S in 2018 was not reinforced. For example, the following categories are examples of those not reinforced in this area: Fast Food, Modern European, Pet Services, Desserts, and Gastropubs. This is an indication that H5, Structure reinforcement, holds in these regions.

We can give a fuller explanation for why this occurs by looking to the most influential groups of new visitors to these neighbourhoods, that is, to the composition of the Tension variable, as defined in Section 4.2.2. As Figure 8(c) indicates, the tracts that are more likely to maintain themselves through time have new visitors (in the Tension variable) that tend to align with the character of the existing Structure. By contrast, in areas (such as Danforth Village) that favour change, new visitors tend to have more incompatible tastes, increasing the tension and reducing the likelihood of mounting effective counter-pressure. This is an indication that H6, Structure-Tension compatibility, holds here. For instance, in the Ossington area in 2011 the average probability values of structure categories with respect to the profiles of the two most influential groups in that area are: Bars $=0.042$, Food $=0.051$, Italian $=0.015$, Nightlife $=0.044$, 
Pizza $=0.01$, Restaurants $=0.117$; being the Alignment value 0.28 . In the same year in the Danforth Village area we have: Food $=0.051$, Hair Salons $=0.002$, Japanese $=0.003$, Restaurants $=0.117$, Shopping $=0.054$, SushiBars $=0.003$; thus, Alignment is 0.23. These examples illustrate the intuition behind H6. Most predominant groups in the Tension in Danforth Village do not align well with the existing Structure. This makes it more likely to see a structural change.

In the following sections, we show that these local cases are illustrative of more general patterns that obtain across all our study areas.

\section{Testing the hypotheses for the entire study area}

Having found some evidence in support of the functional model in a small set of Toronto neighbourhoods, we now examine how well this model generalizes across our entire study area.

\subsection{Equifinality: HI}

Figure 9 shows the distribution of equifinality values for all tracts in all cities. As we can see, in all cities, neighbourhoods over time tend to remain more similar to themselves than to other neighbourhoods. Thus, there is a general tendency toward self-similarity. As discussed, whenever we see this sort of pattern happening in the Homeostatic variable, a functional explanation in which the Homeostatic variable serves as a cause is suggested [19].

Overall, across the six cities under study, we find strong evidence for the equifinality hypothesis. However, it is important to note that there are a few cases where there is a weaker tendency to maintain the Homeostatic variable, i.e., some tracts become more similar to other tracts instead of themselves over the years. All areas are subject to change; however, some change faster and differently than others. According to our hypothesis, these types of areas should not be good candidates for the functional model. 


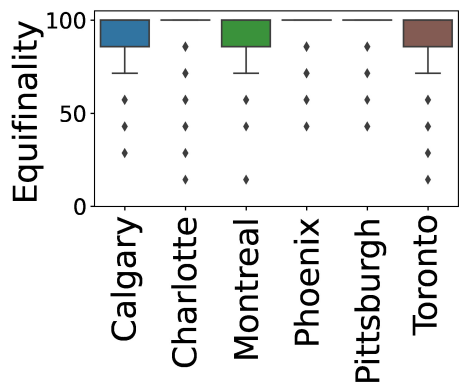

Figure 9: Distribution of equifinality values for all tracts in all cities. Note that most values are 100 in all cases.

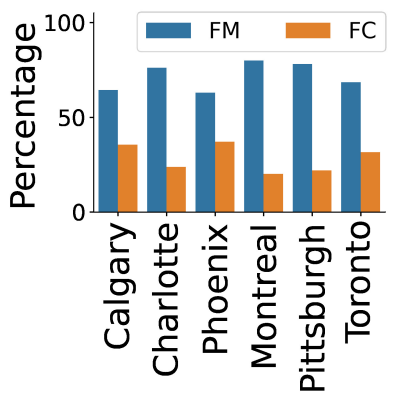

Figure 10: Percentage of FM and FC classes in all cities. [Better in colour]

\subsection{Structural and significant response: $H 2$ and $H 3$}

$\mathrm{H} 2$ and $\mathrm{H} 3$ ask further about the extent to which the conditions for a functional process obtain. Figure 10 presents the number of favour maintenance (FM) and favour change (FC) tracts for each city. Recall that FM tracts represent areas where $\mathrm{H} 2$ and $\mathrm{H} 3$ tend to hold, and FC tracts represent tracts where those hypotheses tend not to obtain. $\mathrm{H} 2$ and $\mathrm{H} 3$ hold considerably in all cities, i.e., the number of FM tracts is much higher than the number of FC tracts. This finding indicates that the Tension-response process characteristic of functional relationships is widespread: where Tensions increase they are generally met by increased Structure activity. H2 and H3 are confirmed. These groups of tracts (FC and FM) are contrasted during the evaluation of the next hypotheses.

\subsection{Homeostatic response: $\mathrm{H} 4$}

H4 tests if neighbourhood self-similarity shows signs of being a homeostatic response to Tensions, specifically if FC tracts show greater changes in their Homeostatic variable $\mathrm{H}$ (e.g. distribution of business types) over time as compared to FM tracts, as described in the Section 4.3.3 Figure 11 presents the distribution of values of tracts' self-dissimilarity for both classes for all cities studied. As we can see, the results indicate that $\mathrm{H} 4$ holds in general, i.e., neighbourhoods of class FC grew more dissimilar from themselves over time than FM neighbourhoods, which showed a greater tendency to reproduce their Homeostatic variable. 


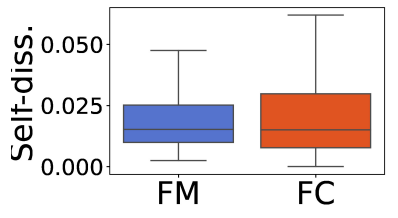

(a) Calgary

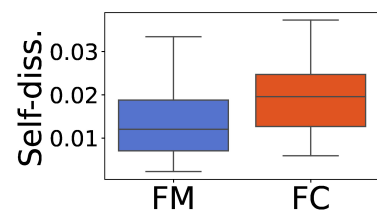

(d) Charlotte

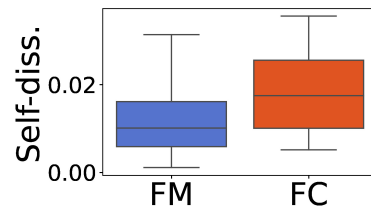

(b) Montreal

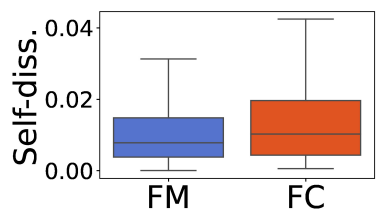

(e) Phoenix

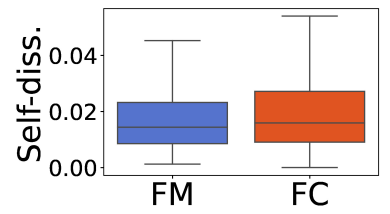

(c) Toronto

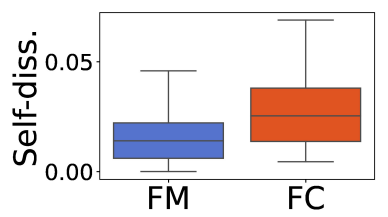

(f) Pittsburgh

Figure 11: Box plots of the distribution of self-dissimilarity values over time for all tracts classified as FM and FC.

\subsection{Structure reinforcement: $H 5$}

H5 tests whether FM areas tend to select or reinforce Structure activities, in contrast to FC areas, where Structure activities should not be reinforced. Figure 12 shows the distributions of the number of categories reinforced (in percentage) for tracts classified as FM and FC. As we can see, all cities have a bigger percentage of categories being reinforced over time in FM tracts. Thus, this is an indication that hypothesis H5, Structure reinforcement, obtains.

\subsection{Alignment of tension's groups and structure: H6}

H6 asks whether neighbourhoods where the functional response holds (FM) tend to receive new visitors (captured in the Tension variable) who show taste profiles similar to those expressed in the existing neighbourhoods' distinctive local business profile (Structure S). Recall that S categories implicitly represent popular categories for regular users of a particular tract. By contrast, where the groups that predominate the Tension variable strongly differ from the taste profile expressed in S, this should correspond to a situation where it is more difficult to maintain the existing identity (FC). 


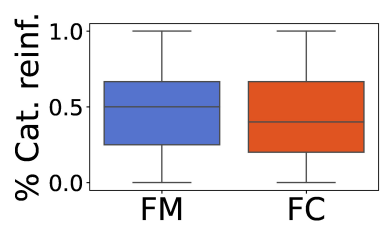

(a) Calgary

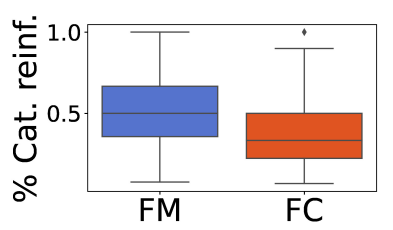

(d) Charlotte

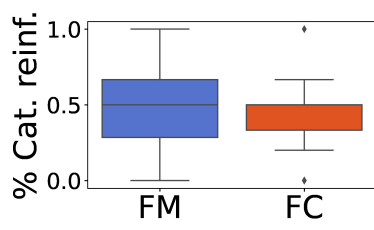

(b) Montreal

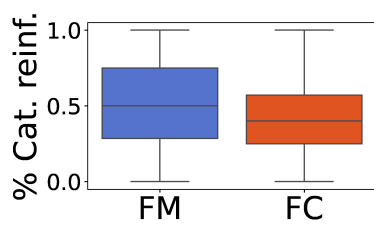

(e) Phoenix

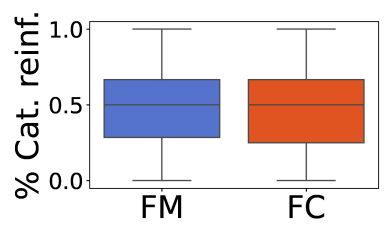

(c) Toronto

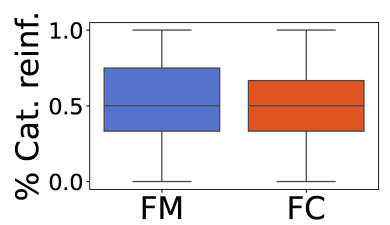

(f) Pittsburgh

Figure 12: Distributions of the number of categories reinforced (in percentage) for tracts classified as FM and FC.

This result is presented in Figure 13 indicating that H6 holds for all cities evaluated; the predominant group tends to be better aligned with the Structure in FM tracts, thus, promoting fewer clashes between Tension and Structures that induce bigger changes in an area. By contrast, in FC tracts, Tension and Structure are less compatible, promoting a stronger clash between new and old.

\section{Discussion and conclusion}

Our study has sought to advance the literature on neighbourhood change and functional explanation by demonstrating how functional explanation can be revived in the context of big data. We adapted the classical model of functional explanation formulated by Stinchcombe to study neighbourhood change with Yelp data. Our formulation explores the complex interactions of geographic and user information to obtain richer information, which is vital for studying the complex causal structures underlying neighbourhood change.

Reviving formal functional explanation is important because it enables common claims in the neighbourhood change literature to be formally tested. For example, with 


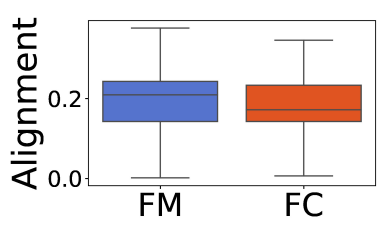

(a) Calgary

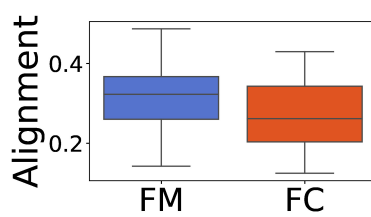

(d) Charlotte

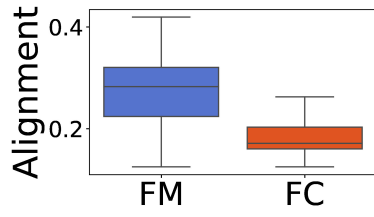

(b) Montreal

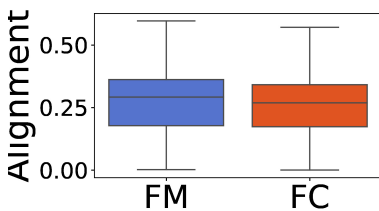

(e) Phoenix

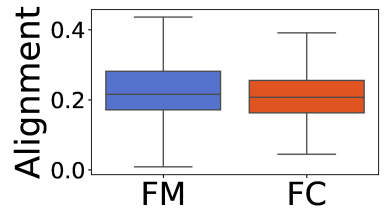

(c) Toronto

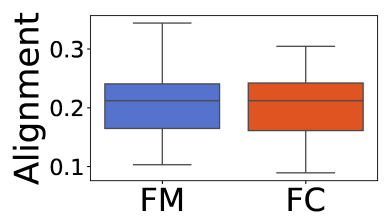

(f) Pittsburgh

Figure 13: Alignment between structure categories and main groups' profiles for tracts classified as FM and FC in all cities.

this model, we can identify the presence or absence of pushback by existing communities against change and evaluate the conditions under which such pushback is more or less likely to succeed. Overall, we showed how to reveal neighbourhood character as a latent tendency to react when met by tensions, expressed in practical actions and reactions rather than only in the content of the local activities or establishments. By proposing and evaluating testable functional hypotheses, we obtained insights into the evaluated variables' contributions to the maintenance of the neighbourhood character or scene.

Our results indicate that the functionalist model assessed could be useful in future explorations. For instance, urban planners can potentially explore it as complementary support in predicting urban change tendencies. This can help create or apply specific urban policies of interest to control or enhance this phenomenon. Future research can also explore other extensions of functional models. For instance, we might search for "functional equivalents" - local amenities or scenes that appear different in content but serve similar functions. This could provide a novel way to classify neighbourhoods by what they do and not just what they are. We could also use functional models to 
identify "structural conflicts" [25], where maintaining the existing neighbourhood state only increases tensions.

Certainly, several extensions could be done as future work. For instance, while we evaluated the choice of parameters manually to favour understanding and explicability, automatic approaches could also be experimented with. Among them, we could perform hyperparameter tuning to determine the optimal values for a given model. Finally, the model could be evaluated using different data sources, such as Foursquare, Google, Facebook, TripAdvisor reviews, or other sources. The main contribution of the present study lies in illustrating methods that could be applied in diverse settings.

Despite the number of significant contributions provided by this study, it is not without limitations. As Spilerman and Gerratana note, while functional explanations provide insights made by the variables studied to the equilibrium of some system, it does not explain its origins [20] (Chapter 11). In our context, we learned, for example, that new groups coming to an area can signal that that area will not maintain its equilibrium - i.e., it will change faster - if there is no appropriate reaction from the Structure. However, our model is silent regarding, for example, why those new groups are being attracted to that area or what makes specific categories popular enough to be part of the Structure. Combining functional and historical explanations is an exciting avenue for further research.

Another limitation is regarding Yelp data. This data source is biased towards a particular user demographic, as is the case for several location-based social networks [59]. Yelp reviewers are relatively equally distributed within the 18-34 and 35-54 age groups (roughly $35 \%$ ), with about $30 \%$ representing the $55+$ age group. Besides, over $60 \%$ are college-educated, and almost half of the users earn $\$ 100,000$ annually, and another $25 \%$ are in the $\$ 60,000-99,000$ range. Information about gender distribution has not been made available by Yelp. However, studies suggest that women represent a slightly greater share of Yelp's user base [60]. Thus, Yelp reviews skew toward the younger, more educated, and middle to high-income users. This means that our results represent partially the Tension and Structures operating in the city. Despite obtaining good results, we believe they can be improved with a less biased dataset, and exploring alternatives is an important area for additional study. 
All in all, this study shows the value of integrating classical social science models and novel data sources for advancing the role of big data in social research.

\section{References}

[1] K. P. Schwirian, Models of neighborhood change, Annual review of sociology 9 (1) (1983) 83-102.

[2] E. v. Beckhoven, G. Bolt, R. v. Kempen, Theories of neighbourhood change and neighbourhood decline: Their significance for post-wwii large housing estates, in: Proc. of ENHR-conference, Reykjavik, Iceland.

[3] G. C. Galster, Making our neighborhoods, making our selves, University of Chicago Press, 2019.

[4] N. L. Prak, H. Priemus, A model for the analysis of the decline of postwar housing, International Journal of Urban and Regional Research 10 (1) (1986) 1-7.

[5] K. Temkin, W. Rohe, Neighborhood change and urban policy, Journal of planning education and research 15 (3) (1996) 159-170.

[6] R. N. Giere, How models are used to represent reality, Philosophy of science 71 (5) (2004) 742-752.

[7] E. C. Delmelle, Mapping the dna of urban neighborhoods: Clustering longitudinal sequences of neighborhood socioeconomic change, Annals of the American Association of Geographers 106 (1) (2016) 36-56.

[8] D. Silver, T. H. Silva, A markov model of urban evolution: Neighbourhood change as a complex process, PLOS ONE 16 (1) (2021) 1-29. doi:10.1371/ journal.pone.0245357

[9] E. L. Glaeser, H. Kim, M. Luca, Nowcasting the local economy: Using yelp data to measure economic activity, Tech. rep., Nat. Bureau of Economic Research (2017). 
[10] M. Somashekhar, Can we bring culture into the large-scale study of gentrification? assessing the possibilities using geodemographic marketing data, Urban Affairs Review 0 (0) (0) 1078087420921459. doi:10.1177/ 1078087420921459

[11] V. Santala, G. Costa, L. Gomes-Jr, T. Gadda, T. H. Silva, On the potential of social media data in urban planning: Findings from the beer street in curitiba, brazil, Planning Practice \& Research 0 (0) (2020) 1-16. doi:10.1080/02697459. 2020.1767394

[12] J. Cranshaw, R. Schwartz, J. Hong, N. Sadeh, The livehoods project: Utilizing social media to understand the dynamics of a city, in: Proc. of ICWSM, Dublin, Ireland, 2012.

[13] T. H. Silva, P. O. V. de Melo, J. M. Almeida, M. Musolesi, A. A. Loureiro, A large-scale study of cultural differences using urban data about eating and drinking preferences, Information Systems 72 (Supplement C) (2017) 95 - 116. doi:https://doi.org/10.1016/j.is.2017.10.002

[14] D. Arribas-Bel, J. Bakens, Use and validation of location-based services in urban research: An example with dutch restaurants, Urban Studies 56 (5) (2019) 868884.

[15] C. Goodwin, The Oak Park strategy: community control of racial change, University of Chicago Press, 1979.

[16] S. Annunziata, C. Rivas-Alonso, Resisting gentrification, in: Handbook of gentrification studies, Edward Elgar Publishing, 2018.

[17] B. Collins, Whose culture, whose neighborhood? fostering and resisting neighborhood change in the multiethnic enclave, Journal of Planning Education and Research 40 (3) (2020) 249-262.

[18] R. J. Sampson, Great American city: Chicago and the enduring neighborhood effect, University of Chicago Press, 2012. 
[19] A. L. Stinchcombe, Constructing social theories, University of Chicago Press, 1987.

[20] A. Gelman, J. Cortina, A quantitative tour of the social sciences, Cambridge University Press, 2009.

[21] K. Davis, The myth of functional analysis as a special method in sociology and anthropology, American sociological review (1959) 757-772.

[22] W. A. Jackson, Functional explanation in economics: a qualified defence, Journal of Economic Methodology 9 (2) (2002) 169-189.

[23] R. K. Merton, Social theory and social structure, Free Press, 1968.

[24] A. Wellstead, M. Howlett, J. Rayner, Structural-functionalism redux: adaptation to climate change and the challenge of a science-driven policy agenda, Critical Policy Studies 11 (4) (2017) 391-410.

[25] G. Arditi, Equilibrium, structural contradictions, and social conflicts: Revisiting stinchcombe, in: Sociological Forum, Vol. 3, Springer, 1988, pp. 282-292.

[26] H. Joas, W. Knöbl, Social theory: twenty introductory lectures, Cambridge University Press, 2009.

[27] M. J. Thompson, A functionalist theory of social domination, Journal of Political Power 6 (2) (2013) 179-199.

[28] J. Vorhaus, Function and functional explanation in social capital theory: A philosophical appraisal, Studies in Philosophy and Education 33 (2) (2014) 185-199.

[29] J. Hackworth, J. Rekers, Ethnic packaging and gentrification: The case of four neighborhoods in toronto, Urban Affairs Review 41 (2) (2005) 211-236.

[30] R. E. Ocejo, The early gentrifier: Weaving a nostalgia narrative on the lower east side, City \& Community 10 (3) (2011) 285-310.

[31] D. Ley, C. Dobson, Are there limits to gentrification? the contexts of impeded gentrification in vancouver, Urban Studies 45 (12) (2008) 2471-2498. 
[32] C. Hamnett, The blind men and the elephant: the explanation of gentrification, Transactions of the institute of British Geographers (1991) 173-189.

[33] P. Eisinger, Reimagining detroit, City \& Community 2 (2) (2003) 85-99.

[34] S. Halebsky, Superstores and the politics of retail development, City \& Community 3 (2) (2004) 115-134.

[35] C. Herring, The new logics of homeless seclusion: Homeless encampments in america's west coast cities, City \& Community 13 (4) (2014) 285-309.

[36] G. Mattson, Urban ethnography's "saloon problem" and its challenge to public sociology, City \& Community 6 (2) (2007) 75-94.

[37] J. Preece, Belonging in working-class neighbourhoods: dis-identification, territorialisation and biographies of people and place, Urban Studies 57 (4) (2020) 827-843.

[38] A. L. Bain, Neighbourhood artistic disaffiliation in hamilton, ontario, canada, Urban Studies 54 (13) (2017) 2935-2954.

[39] H. B. Shin, L. Lees, E. López-Morales, Introduction: Locating gentrification in the global east, Urban Studies 53 (3) (2016) 455-470.

[40] B. L. Ranard, R. M. Werner, T. Antanavicius, H. A. Schwartz, R. J. Smith, Z. F. Meisel, D. A. Asch, L. H. Ungar, R. M. Merchant, Yelp reviews of hospital care can supplement and inform traditional surveys of the patient experience of care, Health Affairs 35 (4) (2016) 697-705.

[41] N. E. Phillips, B. L. Levy, R. J. Sampson, M. L. Small, R. Q. Wang, The social integration of american cities: Network measures of connectedness based on everyday mobility across neighborhoods, Sociological Methods \& Research (2019) 0049124119852386.

[42] K. Joseph, C. H. Tan, K. M. Carley, Beyond local, categories and friends: clustering foursquare users with latent topics, in: Proc. of ACM Ubicomp, ACM, Pittsburgh, USA, 2012, pp. 919-926. 
[43] D. Arribas-Bel, K. Kourtit, P. Nijkamp, The sociocultural sources of urban buzz, Environment and Planning C: Government and Policy 34 (1) (2016) 188-204.

[44] D. Karamshuk, A. Noulas, S. Scellato, V. Nicosia, C. Mascolo, Geo-spotting: mining online location-based services for optimal retail store placement, in: Proc. of ACM KDD, ACM, Chicago, USA, 2013, pp. 793-801.

[45] D. Arribas-Bel, J. Bakens, Use and validation of location-based services in urban research: An example with Dutch restaurants, Urban Studies 56 (5) (2019) 868884.

[46] F. Zhang, L. Wu, D. Zhu, Y. Liu, Social sensing from street-level imagery: A case study in learning spatio-temporal urban mobility patterns, ISPRS Journal of Photogrammetry and Remote Sensing 153 (2019) 48-58.

[47] N. Naik, J. Philipoom, R. Raskar, C. Hidalgo, Streetscore-predicting the perceived safety of one million streetscapes, in: Proc. of the IEEE CVPR Workshops, Columbus, USA, 2014, pp. 779-785.

[48] J.-A. Yang, M.-H. Tsou, C.-T. Jung, C. Allen, B. H. Spitzberg, J. M. Gawron, S.-Y. Han, Social media analytics and research testbed (smart): Exploring spatiotemporal patterns of human dynamics with geo-targeted social media messages, Big Data \& Society 3 (1) (2016) 2053951716652914. doi:10.1177/ 2053951716652914.

[49] S. Zukin, S. Lindeman, L. Hurson, The omnivore's neighborhood? online restaurant reviews, race, and gentrification, Journal of Consumer Culture 17 (3) (2017) $459-479$.

[50] A. W. Olson, F. Calderon-Figueroa, O. Bidian, D. Silver, S. Sanner, Reading the city through its neighbourhoods: Deep text embeddings of yelp reviews as a basis for determining similarity and change, Cities 110 (2021) 103045.

[51] R. R. Nelson, An evolutionary theory of economic change, Harvard university press, 2009. 
[52] D. A. Silver, T. N. Clark, Scenescapes: How qualities of place shape social life, University of Chicago Press, 2016.

[53] Yelp, Yelp dataset, https://www.yelp.com/dataset (2020).

[54] D. M. Blei, A. Y. Ng, M. I. Jordan, Latent dirichlet allocation, Journal of machine Learning research 3 (Jan) (2003) 993-1022.

[55] D. Mimno, H. Wallach, E. Talley, M. Leenders, A. McCallum, Optimizing semantic coherence in topic models, in: Proc. of EMNLP, Edinburgh, UK, 2011, pp. 262-272.

[56] P.-N. Tan, M. Steinbach, V. Kumar, Introduction to data mining, Pearson Education, 2016.

[57] M. Miller, Owner of toronto broth bar responds to accusations of cultural appropriation (2020).

URL https://www.blogto.com/eat_drink/2020/11/ownertoronto-broth-bar-responds-accusations-culturalappropriation/

[58] L. O’Neill, Parkdale steps up protests against vegandale rebranding (2018).

URL https://www.blogto.com/city/2018/08/parkdalebranding-vegandale-toronto

[59] T. H. Silva, A. C. Viana, F. Benevenuto, L. Villas, J. Salles, A. Loureiro, D. Quercia, Urban computing leveraging location-based social network data: A survey, ACM Comput. Surv. 52 (1) (2019) 17:1-17:39. doi:10.1145/3301284.

[60] J. Johnson, Gb: online audience of yelp.co.uk by age group \& gender 2020 (2020).

URL https://www.statista.com/statistics/491925

[61] StatisticsCanada, Canadian business counts (2020).

URL https://www.statcan.gc.ca/eng/start 


\section{Appendix A. Measuring Structure and Tension: robustness checks}

To perform an extra robustness check of our results, we contrasted the score obtained using the original data (best case) with four random scenarios generated on the same data. Random 1: the venues preserve their popularity, and users preserve the number of reviews performed, but the reviews are assigned randomly, simulating users going elsewhere than they actually visited. So, $r_{c_{x}}^{A_{i}, y}$, representing the number of reviews in area $A_{i}$ during $y$, will remain the same, but the users who give those reviews will be random. We apply the same steps performed in the original data in this randomly generated data. Random 2: represents a random distribution of structure peaks. For that, we randomly distribute the observed structure peaks in a certain tract $S P_{i}$. We also ensure that all peaks exist in the random structure, not permitting two random peaks to be assigned to the same position. Random 3: follows the same methodology of Random 2, but with tension peaks instead of structure peaks, i.e., using $T P_{i}$. Random 4: performs Random 2 and 3 simultaneously. All these random processes are repeated 100 times, and the mean and confidence intervals of $99 \%$ of confidence are calculated.

As we can see in Figure A.14 the scores in all random cases, for all cities, were considerably below the score of the original case. This indicates that despite the limitations that our approach has, it captures a phenomenon that is not random.

\section{Appendix B. Canadian business counts vs Yelp}

This section contrasts Yelp data with the Canadian Business Counts - CBC [61]. Figure B.15 shows a comparison of the number of businesses under the category Restaurant in Yelp and the CBC in 2009, 2010, and 2011 for Toronto's neighbourhoods that had at least 50 unique businesses (of any kind) in 2009. As we can see, from 2011, the number of restaurants in both datasets becomes more similar. Besides, only after 2011 there are cases where the number for Toronto surpasses the Census records. This situation is expected to happen because Yelp's classification is provided by users and business owners and Yelp's internal staff, potentially resulting in businesses not being categorized in the same way across the two datasets. For instance, a pub that serves 


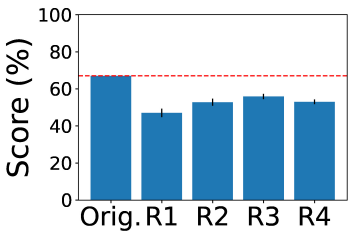

(a) Calgary

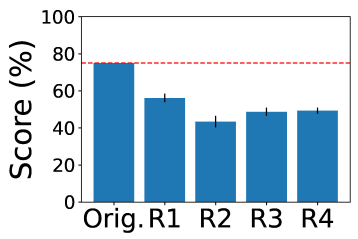

(d) Charlotte

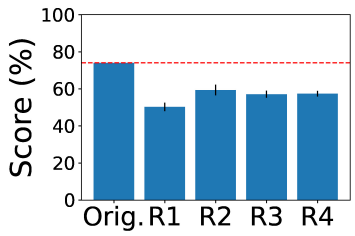

(b) Montreal

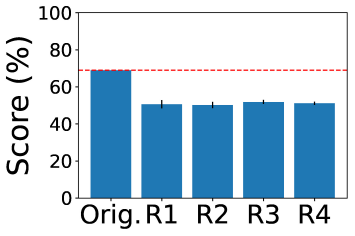

(e) Phoenix

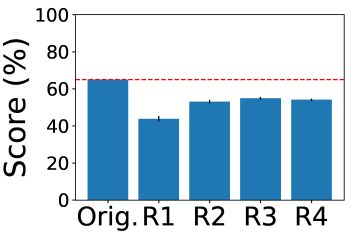

(c) Toronto

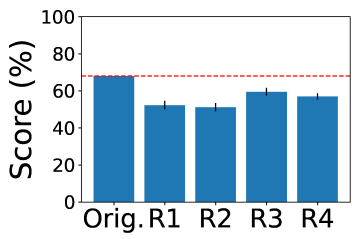

(f) Pittsburgh

Figure A.14: Original score compared to four random cases: Random 1 (R1) - simulating users going to other places, the venues preserve their popularity, but the users are random; Random 2 (R2) - representing shuffled structure values before the analysis; Random 3 (R3) - representing shuffled tension values before the analysis; and Random 4 (R4) - representing shuffled structure and tension values before the analysis.

food might be classified as a "drinking place" in CBC, while in Yelp, it could be classified as a pub and restaurant. Also, Yelp includes restaurants with no employees, and this is not the case for CBC.

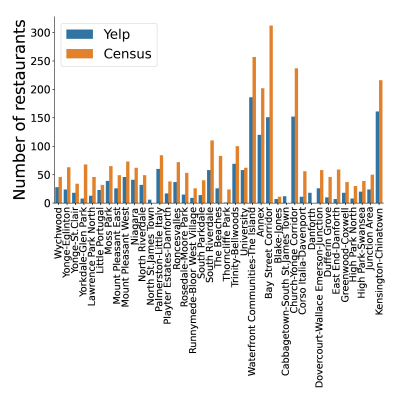

(a) 2009

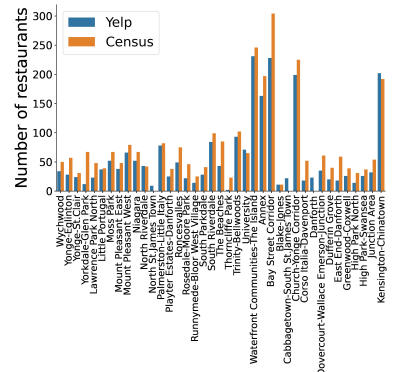

(b) 2010

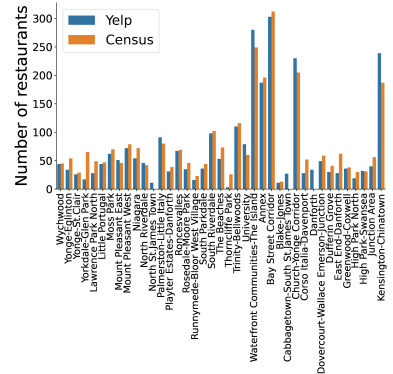

(c) 2011

Figure B.15: Number of businesses under the category Restaurant in Yelp and in the Canadian Business Counts in Toronto's neighbourhoods. [Better in colour] 